\title{
Can regional spreading of amyotrophic lateral sclerosis motor symptoms be explained by prion-like propagation?
}

\author{
Tadashi Kanouchi, ${ }^{1}$ Takuya Ohkubo, ${ }^{2}$ Takanori Yokota ${ }^{2}$
}

\begin{abstract}
'Department of Clinical Laboratory, Tokyo Medical and Dental University, Tokyo, Japan ${ }^{2}$ Department of Neurology and Neurological Science, Tokyo Medical and Dental University, Tokyo, Japan
\end{abstract}

Correspondence to Professor T Yokota, Department of Neurology and Neurological Science, Tokyo Medical and Dental University, 1-5-45 Yushima, Bunkyo-ku, Tokyo 113-819, Japan;

tak-yokota.nuro@tmd.ac.jp

Received 10 November 2011 Revised 19 March 2012 Accepted 20 March 2012 Published Online First 27 April 2012

\section{SUNLOCK:D}

This paper is freely available online under the BMJ Journals unlocked scheme, see http:// jnnp.bmj.com/site/about/ unlocked.xhtml

\section{ABSTRACT}

Progressive accumulation of specific misfolded protein is a defining feature of amyotrophic lateral sclerosis (ALS), similarly seen in Alzheimer disease, Parkinson disease, Huntington disease and Creutzfeldt-Jakob disease. The intercellular transfer of inclusions made of tau, $\alpha$-synuclein and huntingtin has been demonstrated, revealing the existence of mechanisms reminiscent of those by which prions spread through the nervous system. Evidence for such a prion-like propagation mechanism has now spread to the major misfolded proteins, superoxide dismutase 1 (SOD1) and the $43 \mathrm{kDa}$ transactive response DNA binding protein (TDP-43), implicated in ALS. The focus in this review is on what is known about ALS progression in terms of clinical as well as molecular aspects. Furthermore, the concept of 'propagation' is dissected into contiguous and non-contiguous types, and this concept is expanded to the severity of the focal symptom as well as its regional spread which can be explained by cell to cell propagation in the local neuron pool.

\section{INTRODUCTION}

Amyotrophic lateral sclerosis (ALS) is a fatal neurodegenerative disease characterised by loss of motor neurons in the motor cortex, brainstem and spinal cord. ALS appears to start at a focal body region and then muscle paralysis spreads to other regions over time. Eventually both the upper (UMNs) and lower (LMNs) motor neurons are diffusely involved, and the paralysis ultimately causes death due to respiratory failure. Approximately $90 \%$ of all cases are classified as sporadic ALS, defined as having no family history of the disease. The remaining cases, designated familial ALS, are inherited in a dominant or recessive fashion. Of the known 13 genes, mutations in superoxide dismutase 1 (SOD1), the $43 \mathrm{kDa}$ transactive response DNA binding protein (TDP-43), fusion in sarcoma, angigenin and optineurin cause the typical ALS phenotype. ${ }^{1}$ Cytoplasmic or nuclear inclusions are one histopathological characteristic of sporadic ALS. A breakthrough in understanding sporadic ALS pathogenesis was the discovery of TDP-43, which was identified as the major component of the protein aggregates and of the insoluble fraction of the brains of patients with sporadic ALS and frontotemporal lobar degeneration. ${ }^{2}{ }^{3}$ TDP-43 is now presumed to play an essential role in the pathogenesis of sporadic ALS, possibly equivalent to that of $\tau$ and $\beta$ amyloid in Alzheimer's disease or $\alpha$-synuclein in Parkinson's disease.
These pathogenic proteins or peptides have common features: they are misfolded, they selfaggregate and they form inclusions with cross$\beta$ conformation and possibly toxic function. Recent research has shown a remarkable new concept, 'prion-like propagation,' in non-infectious neurodegenerative diseases, in which pathogenic proteins cross cellular membranes and are excreted into the extracellular space where they are transferred to adjacent cells; this is similar to the behaviour of the characteristic pathogenic prion protein (PrPsc). This intercellular transfer of pathogenic proteins leads to the neuropathological spread of the lesion.

This article highlights the basic and clinical aspects of the pathological progression of ALS and discusses the possible mechanism of 'prion-like propagation' in the regional spread of ALS symptoms.

\section{DISEASE ONSET AND PROGRESSION IN ALS}

Disease onset and disease progression are thought to have a different mechanism. Because the motor symptoms of ALS are usually initiated in one or two highly localised sites, the motor symptoms and the responsible lesions always spread regionally as the disease progresses. Many neurologists have thought that regional spread of the symptoms is just one aspect of disease progression in ALS because ALS is a systemic disease that eventually involves the entire UMN and LMN systems, and every motor neuron in ALS ubiquitously has a cause of disease. The most easily understood example is familial ALS with mutations in SOD1 or TDP-43, in which every motor neuron and glial cell has a chromosomal genetic defect that causes motoneuronal cell death (figure $1 \mathrm{~A}-\mathrm{a}$ ). For the initial onset, in sporadic as well as familial ALS, molecular change most likely predates clinical onset. A motor neuron becomes symptomatic when the accumulated molecular pathology, including increased pathogenic protein aggregates, exceeds a certain threshold. In sporadic cases, the multifocal hit hypothesis has a different mechanism but represents a similar phenotype in which an acquired defect in chromatin, DNA, RNA or proteins (such as epigenetic alternation, a somatic DNA mutation, RNA editing error or misfolding of proteins possibly due to ageing effects or various environmental stressors) occurs randomly in each cell, and these defects accumulate and converge to initiate the pathogenic process (figure $1 \mathrm{~A}-\mathrm{b}$ ). The multifocal hit hypothesis (figure $1 \mathrm{~A}-\mathrm{b}$ ) is different 
A Onset mechanism/ non-propagative progression mechanism
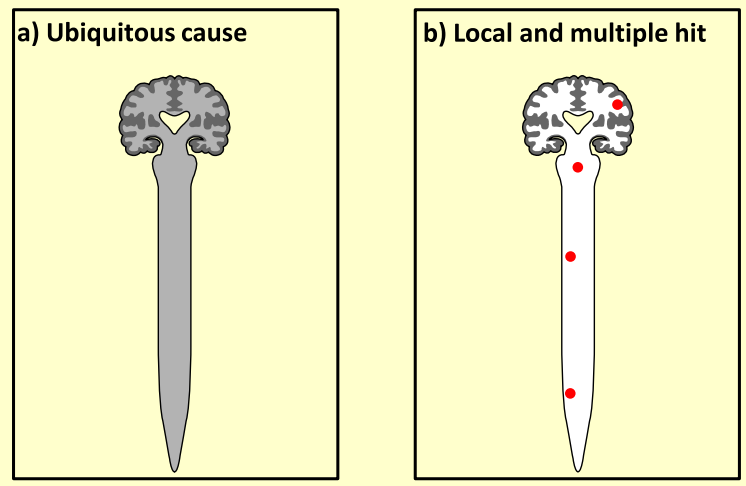

B Propagative progression mechanism
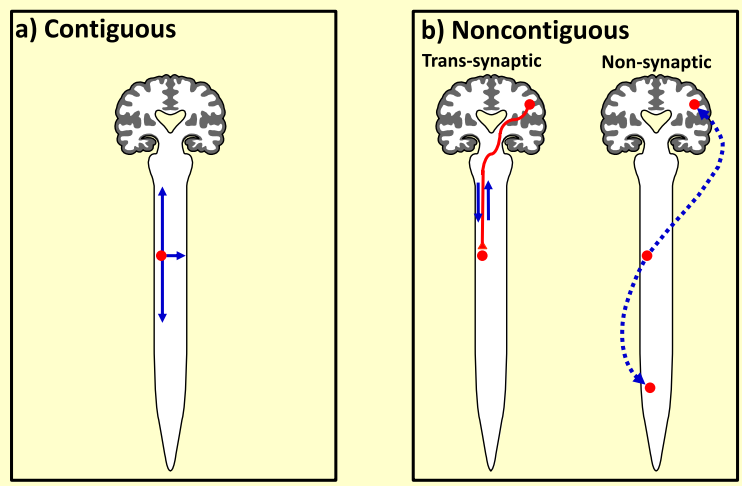

Figure 1 Onset and regional spread mechanisms of amyotrophic lateral sclerosis (ALS) lesion. Symptom is initiated by focal neuronal change, the onset mechanism of which exists in every motor neuron $(A-a)$, or is limited to the affected neuron $(A-b)$. Regional spread of symptoms may be caused by summation of the increased number of the symptomatic or hit neurons. If the spread mechanism of ALS lesion is explained by propagation, it is classified as local and contiguous type $(B-a)$ or remote and non-contiguous type $(B-b)$. The contiguous type propagation is based on cell to cell transfer of disease property between the neurons proximate to each other and has a radial and horizontal vector of spread. The non-contiguous, trans-synaptic type propagation includes anterograde spread (dying forward) or retrograde degeneration (dying back) of neural networks. The non-contiguous non-synaptic type propagation includes remote transfer of the toxic molecule through blood and CSF.

from the ubiquitous change mechanism (figure $1 \mathrm{~A}-\mathrm{a}$ ) in that the molecular change occurs in individual cells, and unaffected cells do not have any molecular defect. In both mechanisms, the region of initiation may be determined by a stochastic hit but there is a vulnerability among the motoneurons resulting in more frequent focal involvement of the tongue and distal limb muscles in the early stage. These two schemas shown in figure $1 \mathrm{~A}-\mathrm{a}$ and figure $1 \mathrm{~A}-\mathrm{b}$ are a possible onset mechanism of ALS but the regional spread of the motor symptoms as a disease progression can also be explained by just summation of the increased number of symptomatic or hit neurons by the same mechanism without following propagative pathology.

The concept of 'prion-like propagation' is the intercellular transmission of the disease phenotype, typically considered to be mediated by an aggregate of pathogenic proteins. Local contiguous spread could occur through cell to cell 'domino-like' propagation between neighbouring neurons in a cell autono- mous or non-cell autonomous manner (figure $1 \mathrm{~B}-\mathrm{a}$ ). In the case of spinal motor neurons, this local and contiguous spread has two vectors in the three-dimensional anatomy of motoneurons determined by its anatomical structure: one is radial, leading to rostral or caudal spread in the motor neuron column beyond the spinal segment, and the other is lateral/horizontal, leading to lateral to medial spread on one side of the anterior horn showing progression from distal to axial muscles, and contralateral spread within the same spinal segment showing progression to the contralateral limb (figure $1 \mathrm{~B}-\mathrm{a}$ ). ${ }^{4}$

If the spread is non-contiguous, there are two possible mechanisms: (1) anterograde trans-synaptic spread (dying forward) or retrograde degeneration (dying back) of neural networks (figure $1 \mathrm{~B}-\mathrm{b}$, left); and (2) non-synaptic and remote spread of a misfolded protein or toxic molecule through the blood or CSF via exosomes, similar to the metastasis of cancer cells (figure $1 \mathrm{~B}-\mathrm{b}$, right). ${ }^{5}$ Before regional propagation can occur, there must be a focal lesion that develops with a molecular mechanism that is different from that of propagation. This initiation mechanism should include ubiquitous (figure $1 \mathrm{~A}-\mathrm{a}$ ) or local and multiple hit (figure $1 \mathrm{~A}-\mathrm{b}$ ) pathology. The contiguous and non-contiguous propagation mechanisms are not independent but can coexist with each other, and therefore the final clinical picture appears to be a complex combination of single or multiple hits, with or without contiguous or non-contiguous propagation.

Disease progression in ALS consists of regional spread of motor symptoms and exacerbation of local motor symptoms, such as a decrease in grip strength and progression of thenar muscle atrophy. We suppose that such an exacerbation of focal motor symptoms is also explained by the contiguous propagation mechanism. For example, the severity of hand muscle atrophy is determined by the remaining number of motor neurons in the lateral nuclear group of the spinal anterior horn innervating the hand muscle after neuronal degeneration and reinnervation of the denervated muscle fibres. In this motor neuron pool of the lateral nuclear group, neuronal degeneration might be mediated by local cell to cell propagation of the disease property between spinal motor neurons proximate to each other after the seeding to a single motor neuron (figure 2). This indicates that the concept of 'propagation' can include exacerbation of the focal symptom as well as its regional spread.

\section{MOLECULAR BASIS OF PROPAGATION IN ALS}

Protein to protein interaction, seeded aggregation and propagation in ALS

Familial ALS is classified into two groups, with and without aggregation of TDP-43. The cytoplasmic mislocalisation and aggregation of TDP-43 is now the major pathological hallmark for sporadic ALS. Similar TDP-43 pathology is found in familial ALS patients with mutation of optineurin, C9ORF72 and VCP, but is not observed in those with mutation of SOD1 and FUS. In the past 2 years, there have been cumulative data that normal and mutant SOD1 and normal TDP-43 can undergo a similar seeded aggregation, ${ }^{6}$ although the pathophysiology of TDP-43 and mutant SOD1 is different and difficult to discuss in the same platform.

TDP-43 is a nuclear protein and consists of two RNA recognition motifs (RRM1 and RRM2) and a proteolytic C terminal domain, where most of the mutations are found. $\mathrm{C}$ terminal fragments of TDP-43 have been identified in the cytoplasmic inclusions of sporadic ALS patients. ${ }^{2} 3$ TDP-43 readily aggregate in vitro which is enhanced by ALS associated mutation, ${ }^{7}$ and $C$ terminal fragments may play a critical role in TDP-43 


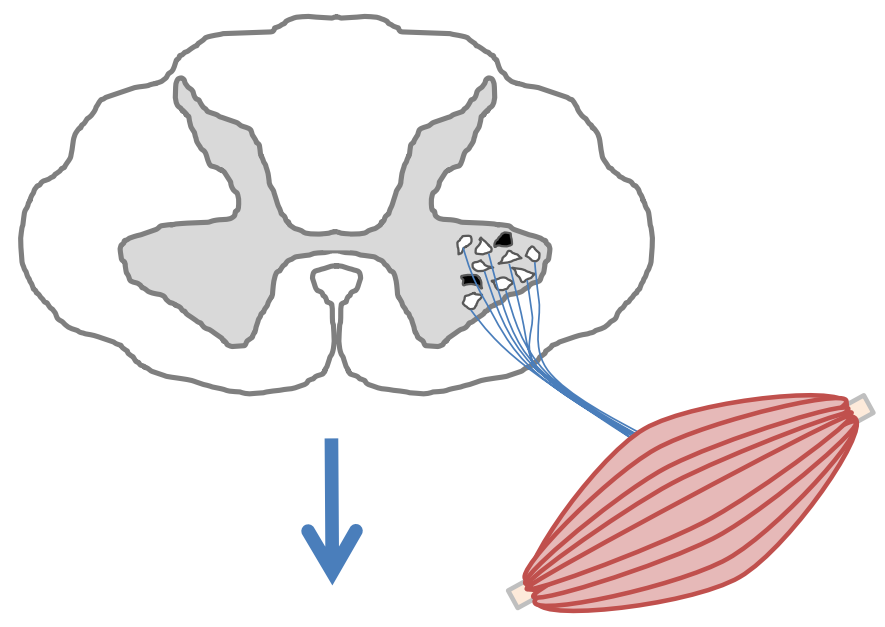

membrane. Exosomes are small lipid membranous microvesicles which are usually formed by the fusion of multivesicular bodies with the plasma membrane. The biologically important functions of exosomal release are the secretion of proteins in order to remove unwanted proteins, the delivery of signals to other cells and the transfer of pathogens among cells. Exosomes derived from prion infected neuronal cell lines are efficient initiators of prion propagation in uninfected recipient cells and non-neuronal cells. Indeed, mutant SOD1 has been proposed to be actively secreted with neurosecretory vesicles through an abnormal interaction with chromogranins $\mathrm{A}$ and $\mathrm{B} .{ }^{12}$ Protein aggregates extracted from mutant SOD1 expressed cells could penetrate into cells by macropinocytosis. ${ }^{6}$ Importantly, a passive immunisation approach based on intracerebroventricular infusion in G93A-SOD1 mice of monoclonal antibodies specific to misfolded forms of SOD1 succeeded in reducing the level of mutant SOD1 in the spinal cord and in prolonging the lifespan of G93A-SOD1 mice to a minor degree. ${ }^{13}$ The exact mechanisms by which antiSOD1 antibodies confer protection are not yet fully understood but the toxic effect of the excreted mutant SOD1 might be blocked by the antibodies.

\section{Glia to neuron propagation of toxicity in ALS}

Toxic factors secreted or released from microglia and astrocytes damage motor neurons in ALS mouse models of mutant SOD1, which may lead to disease progression. ${ }^{14}{ }^{15}$ In vivo transplanted glial restricted precursor cells expressing mutant human SOD1 (G93A) survive and differentiate efficiently into astrocytes in the spinal cords of mice, and graft derived mutant human SOD1 astrocytes induce ubiquitination and death in host motor neurons. Only mutant human SOD1 expressing astrocytes can induce wild-type motor neuron death in mice. ${ }^{16}$ Conversely, siRNA knockdown of SOD1 in astrocytes significantly attenuates toxicity against motor neurons. In ALS, astrocytes might induce and regulate unknown toxic factors that have a non-cell

Figure 2 The severity of the focal symptom can be explained by local propagation. Exacerbation of hand muscle atrophy is caused by loss of $\alpha$-motor neurons in the motor neuron pool in the spinal anterior horn innervating the hand muscle. Such a severity of the focal symptom also may be mediated by local cell to cell propagation between neighbouring spinal motor neurons.

aggregation. $^{8} 9$ In cultured cells, misfolded forms of TDP-43 can initiate intracellular aggregation and induce the misfolding and subsequent aggregation of the respective native proteins. ${ }^{8} 9$ What triggers TDP-43 aggregation has not been determined but increased protein concentration due to disruption of microtubule transport ${ }^{9}$ or chronic cellular stress and defects in stress granule disassembly ${ }^{10}$ occurring with the ageing process may converse to its initiation of this pathological event.

SOD1 is a stable dimer and is highly resistant to proteolytic degradation. More than 150 point mutations have been identified in SOD1, occurring at almost every position; these might impair the stability of the protein and influence the formation of aggregates and inclusion bodies. Both native and mutant forms of SOD1 associated with each other, and some types of mutant SOD1, easily form aggregates and fibrils under denaturing conditions. ${ }^{5} 611$ Importantly, removal of the misfolded seeds does not stop aggregation of endogenous SOD1, indicating that the newly formed aggregates can act as templates for the subsequent misfolding of additional native SOD1. ${ }^{11}$

\section{Neuron to neuron propagation in ALS}

Cellular proteins could be released from neurons via vesicle mediated exocytosis or direct leakage through a damaged cell 
having left-sided onset. In contrast, no predominance for side or footedness has been found in patients with lower limb onset. These findings suggest that frequent use may influence symptom onset because the dominant hand will be used more frequently and both legs will be almost equally used in daily activity although a case control study revealed no association between physical activity and the risk of developing ALS. ${ }^{24}$ The corticomotoneuronal organisation in the dominant and nondominant hand could also explain the difference in right and left onset.

Different motor neurons may also have different vulnerabilities. In a three-dimensional study of the distribution of anterior horn cells in the cervical eighth segment of the human spinal cord obtained at autopsy from patients with ALS, zones of focal and patchy loss of motor neurons were identified without consistent severity or distribution at different sectional levels. ${ }^{25}$ According to the investigators, these findings imply that different pools of motor neurons have varying resistance to disease. In limb onset cases, the initial symptoms present most commonly in distal muscles. In an analysis of 1200 patients, Haverkamp et $a l^{22}$ found that $73 \%$ of non-bulbar patients first exhibited symptoms in the distal portions of the limbs. In 24 autopsies of ALS patients, Tsukagoshi et $a^{26}$ found more marked anterior horn cell loss at the eighth cervical segment, which mainly innervates the distal hand muscles, than at the sixth cervical segment innervating more proximal upper limb muscles. The distal predominance in the initial symptoms does not necessarily reflect only axon length dependent vulnerability because bulbar and lower limb onset occur at the same frequency. By using quantitative concentric needle EMG in the early stages of ALS, de Carvalho et $a l^{27}$ showed that similar abnormalities in motor unit potentials (MUPs) were found in paraspinal and limb muscles in the same spinal segment, both in the sixth cervical segment and the fifth lumbar segment, although fasciculation potentials were more frequent in limb muscles than in paraspinal muscles, and fibrillations were most frequent in the distal tibialis anterior muscle. They concluded that the results are consistent with generalised involvement of motor neurons in the spinal segments in the early stages of ALS progression. The vulnerability might differ not only in motor neurons themselves but also in the segments or sites within a segment.

Another vulnerability may depend on axonal excitability. In ALS, muscle wasting preferentially affects the thenar complex, including the abductor pollicis brevis and first dorsal interosseous (FDI) muscles, compared with the hypothenar muscle (abductor digiti minimi (ADM)). This peculiar pattern of dissociated atrophy of the intrinsic hand muscles, termed the 'split hand', is nearly specific in ALS. ${ }^{28}$ Using threshold tracking, Bae et $a^{29}$ investigated the different membrane properties of FDI and ADM motor axons in the same ulnar nerve at the same site in 12 normal subjects, and demonstrated that nodal persistent sodium conductances are more prominent in FDI axons than in $\mathrm{ADM}$ axons, and therefore excitability is physiologically higher in FDI axons. The higher excitability in FDI axons than in ADM axons may explain the preferential involvement of FDI over ADM in ALS.

There can also be selective vulnerability by physiological subtypes of motoneurons. Pun et al ${ }^{30}$ showed in two mouse models of motoneuron disease (G93A SOD1 and G85R SOD1) that axons of fast fatigable motoneurons were affected synchronously, long before symptoms appeared, and fast fatigue resistant motoneuron axons were affected at symptom onset, whereas axons of slow motoneurons were resistant. In later stages of human sporadic ALS, motor units with high recruitment thresholds for voluntary contraction, which correspond to the larger motoneurons, were more affected in patients with more severely affected FDI muscles. ${ }^{31}$

\section{Spreading patterns of clinical manifestations}

In the progression of ALS, muscle weakness regionally spreads over time, and most UMNs and LMNs ultimately degenerate. Spread of clinical manifestation, as well as exacerbation of focal motor symptom, should reflect an increase in the number of affected UMNs and LMNs although disturbance in a given motoneuron can have widespread effects because of synaptic dysfunction and loss of control at a distance in large and complex neural networks. If cell to cell propagation of disease property would make healthy adjacent motoneurons ill, the simplest pattern of symptom spread should be the contiguous spread into adjacent body regions. However, although spread is contiguous in many ALS patients, it is sometimes non-contiguous.

Ravits et al ${ }^{1932}$ described, from cross sectional clinical observations, that the focal and discrete motor manifestations of ALS present at clinical onset spread outward into contiguous body regions. In 14 of 19 ALS nervous systems examined at autopsy, the regional lower neuron loss within the nervous system was graded radially away from the region of onset, suggesting contiguous spreading of the degenerative process. ${ }^{33}$ This contiguous spreading pattern has also been found by other investigators who observed cohorts of ALS patients longitudinally. ${ }^{20}{ }^{21}$ Horizontal spreading along the neuroaxis occurs more frequently than radial spreading-that is, the contralateral homologous limb tends to be involved sooner than other regions in patients with unilateral limb onset ${ }^{18} 2034$ although such a directionality was not observed in one study. ${ }^{35}$ In patients with bulbar onset, radial spreading to the upper limbs occurred sooner than spreading to the lower limbs in one study ${ }^{21}$ although the difference was not statistically significant in another study. ${ }^{18}$ These observations suggest that one factor influencing the time to symptomatic spread could be distance from the onset site. Another important feature of radial spreading is the preferential involvement of the side ipsilateral to the side of onset-that is, the leg ipsilateral to the onset arm and the arm ipsilateral to the onset leg tend to be involved before the contralateral limbs. ${ }^{18} 2034$ The underlying mechanisms of the preferential ipsilateral spread have not yet been elucidated but radial propagation on the ipsilateral side or the neural connections projecting to the ipsilateral cervical and lumbar spinal motoneurons, such as the corticospinal system, might be involved.

Spread to non-contiguous regions has also been documented although less frequently than contiguous spreading. FujimuraKiyono et al ${ }^{21}$ showed that, among 150 patients with sporadic ALS who underwent follow-up every 3 months, the second region involved was a distant (non-contiguous) region in $14 \%$ of 52 patients with lower limb onset and in $29 \%$ of 32 patients with bulbar onset. A non-contiguous spread pattern was also found in $14.8 \%$ of 282 sporadic ALS patients investigated retrospectively by Gargiulo-Monachelli et al ${ }^{35}$ If ALS is a disease whose pathological process starts as a focal 'seed' and then propagates outward, the non-contiguous spreading pattern implies that some cases start in multiple foci. This possibility is supported by the existence of patients with clinical onset in two regions at almost the same time. Such cases were found in $10.6 \%$ of a cohort of 150 patients with sporadic ALS. ${ }^{21}$ Körner et al ${ }^{20}$ also found patients with lower limb onset who already had bulbar involvement at disease onset. Among 19 autopsy cases of 
sporadic ALS, the loss of motoneurons was greatest in a region different than that identified as the region of clinical onset in two cases. ${ }^{33}$ If only one seed is involved, one possible explanation for non-contiguous spreading is anterograde trans-synaptic propagation. Supporting this, the spread between bulbar and spinal regions occurs preferentially in the rostral-caudal direction than caudal-rostral direction ${ }^{18-21}$ although this difference was not obvious in one study. ${ }^{35}$ Assessments of the cumulative occurrence of symptomatic involvement, based on information from serial questionnaires, demonstrated that limb symptom accrual in 155 bulbar onset ALS patients was significantly faster than bulbar symptom accrual in 387 ALS patients with unilateral limb onset over 6 years of follow-up. ${ }^{18}$ On Kaplan-Meier plots, duration from bulbar onset to limb involvement was shorter than the reverse $(9$ months from bulbar regions to upper limb vs 17 months from upper limb to bulbar regions and 14 months from bulbar regions to lower limb vs 27 months from lower limb to bulbar regions until occurrence in $50 \%$ of patients). ${ }^{21}$ In the monkey, it was demonstrated that some pyramidal tract neurons in the hand and trunk areas of the primary motor cortex have multiple axon branches projecting to motor neuron pools in non-adjacent spinal segments. ${ }^{36}$

However, it is important to note that the degenerative process advances insidiously, and at least one-third of LMNs for a given muscle were estimated to be lost before the clinical signs manifest. Indeed, widespread abnormalities on needle electromyography are often found even if clinical deficit is localised, indicating that the clinical symptom manifests according to the balance between the rate of motoneuron loss and the ability of collateral sprouting of the remaining motoneurons which compensates the loss. Therefore, intensive needle electromyographic studies are needed for the analysis of non-contiguous spreading.

\section{Relation between upper and lower motor neuron involvement}

In ALS, neurodegeneration occurs in both the UMNs in the cortex and the LMNs in the brainstem and spinal cord, and a variable combination of $\mathrm{UMN}$ and $\mathrm{LMN}$ involvement produces a wide variety of motor symptoms. However, the relationship between UMN and LMN involvement is not yet fully clear. There is ongoing discussion as to whether the UMNs or LMNs are primarily involved (anterograde 'dying forward' transneuronal degeneration vs retrograde 'dying back' degeneration) or each is independently affected.

With regard to the onset site, Ravits et al ${ }^{32}$ reported that during the early stages of ALS, UMN and LMN signs were maximal in the same peripheral body region. They speculated that the triggering pathogenic event may be distributed by transneuronal signalling or axonal transport, which is relevant to the trigger and initial distribution between UMNs and LMNs. In contrast, Körner et $a l^{20}$ did not find a correspondence of onset regions between UMNs and LMNs in their cohort. They found that the frequency of UMN signs was independent of the onset region and was highest in the proximal legs at any given time although the side of involvement corresponded with the UMN and LMN signs. They concluded that the UMNs of the lumbar region are more vulnerable.

With regard to regional spread, Ravits et al found that the outward spread of UMN and LMN signs differed during disease progression; these signs either spread to different peripheral body regions or to the same peripheral body region but to different extents, and there was no correlation in the severity of UMN and LMN involvement. Other neuropathological and neurophysiological findings seem to be consistent with the independent involvement of UMNs and LMNs. In 12 autopsy cases of the classical sporadic form of ALS, the pyramidal cells in layer 5 of the tongue and foot areas of the precentral gyri were significantly smaller in patients than in controls but no correlation was found between the mean sizes of pyramidal cells and the numbers of surviving LMNs in the hypoglossal nucleus or in the anterior horn of the fourth lumbar segment of the spinal cord. ${ }^{37}$ In 18 cases of sporadic motor neuron disease, there was poor and negative correlation between the densities of the spinal motor neurons in the 6th-8th cervical segments and of the corticomotoneurons in the corresponding areas. ${ }^{38}$ In a longitudinal neurophysiological study, Attarian et al ${ }^{39}$ investigated the changes over time of MUPs in the extensor carpi radialis and the same single motor unit responses induced by transcranial magnetic stimulation (TMS). They found no correlation between UMN and LMN dysfunction in the course of disease progression. A positive correlation was observed between macroMUP size and the amplitude of the excitatory responses to TMS only in the earliest stage of the disease but this correlation disappeared within the second year after onset. Analysis of MUPs in the sternomastoid, which receives both contralateral and ipsilateral corticospinal innervation, and in the trapezius, which usually only receives contralateral innervation, revealed symmetric changes in both muscles, and the two muscles were equally affected, suggesting that UMNs and $\mathrm{LMNs}$ are independently involved, given the characteristic asymmetry of ALS. $^{40}$

In contrast, Körner et $a l^{20}$ observed that UMN and LMN signs predominantly spread together, rather than independently, as Ravits et al described. In the Körner et al study, LMN involvement was more distinctive than UMN involvement at disease onset, which may support the hypothesis of initial involvement of LMNs with a retrograde 'dying back' process leading to secondary UMN degeneration.

On the other hand, the findings from a study using TMS in patients with familial ALS with SOD1 mutations could support the hypothesis of initial involvement of UMNs with an anterograde 'dying forward' transneuronal process leading to secondary LMN degeneration. Cortical hyperexcitability, which has been shown in the early stage of sporadic ALS, ${ }^{41}{ }^{42}$ was also found in three presymptomatic SOD1 mutation carriers, and subsequently these three individuals developed the clinical features of ALS. ${ }^{43}$ Loss of inhibitory cortical interneurons is assigned as one possible mechanism for such cortical hyperexcitability in the early stage of the disease. These cells may be the earliest to be involved. The increased concentration of extracellular glutamate may also cause the hyperexcitability of corticospinal neurons.

Interestingly, the split hand in ALS may have a cortical basis. ${ }^{44}$ A study using TMS demonstrated that the ratios of motor evoked potentials to compound muscle action potential were significantly larger for the thenar complex in normal subjects. This suggests there is a stronger corticomotoneuronal input to these muscles, and relatively extensive corticomotoneuronal inputs on the thenar spinal anterior horn cells would be associated with more glutamate release, which may cause preferential degeneration in ALS through glutamate induced excitotoxicity. A similar rationale might exist for the preferential involvement of the dominant hand in ALS. A TMS study in relation to handedness in normal subjects demonstrated a shorter cortical silent period, reflecting reduced inhibitory function, in the dominant hand. ${ }^{45}$ The corticomotoneuronal organisation might be relevant to the vulnerability of LMNs rather than to the propagation of pathogenic molecular events. 


\section{FUTURE STUDIES AND THERAPEUTIC IMPLICATIONS OF PROPAGATION IN ALS}

We do not have any concrete clinical or in vivo biological evidence of propagation in ALS progression. Several longitudinal and cross sectional analyses of clinical manifestations tell us that the regional spreading pattern is contiguous in many ALS patients, implying that propagation does contribute to progression of this disease. On the other hand, up to $29 \%$ of patients show a 'skipping' pattern of spread, such as lower limb manifestations following bulbar onset. This is partly because UMN and LMN signs, even if they are correlated, often progress differently. Moreover, all of these reports were based on studies of the involvement of broad body regions, such as the bulbar region and upper and lower limbs, and the continuity of lesions in spinal segments or motor cortical topography were not evaluated. In the spinal lesions, most neurologists do not consider that all ALS lesions are contiguous because muscle atrophy is frequently observed in distal hand and foot muscles. ${ }^{20} 22$ To make the story more complex, motoneurons have differing vulnerabilities to ALS pathology, and the concept of 'propagation' includes non-contiguous spread.

To clarify how ALS progresses, we need to determine in ALS patients whether single or multiple spinal and cortical lesions are present in the early stages, and whether these lesions progress contiguously or non-contiguously. Prospective and longitudinal investigations with detailed electrophysiological and advanced neuroimaging analyses are needed to address these questions. In basic research, we need a cellular model for identifying the transferred molecule and for dissecting the molecular mechanism of cellular propagation, and an animal model for analysing the spatiotemporal spread of ALS lesions.

Nonetheless, the hypothesis of propagation could have important therapeutic implications. We expect that the mechanism of disease onset is different from that of progression, and therefore therapy for the cause of disease onset will not necessarily be enough to cure ALS patients who are already symptomatic. The development of effective treatments will depend on elucidation of the specific molecular mechanisms of progression, and if focality is truly a feature of ALS, then therapies could be applied regionally at early stages to suppress or prevent progression and to spare the critical neurons that control respiration.

\section{Acknowledgements The authors thank Miss Mio Tajiri for her help.}

Contributors All of the authors made substantial contributions to the conception and design of this review article. The authors drafted the article together and revised it critically for important intellectual content. The version to be published was approved by all of the authors.

\section{Competing interests None.}

Provenance and peer review Commissioned; externally peer reviewed.

\section{REFERENCES}

1. Kiernan M, Vucic S, Cheah B, et al. Amyotrophic lateral sclerosis. Lancet 2011:377:942-55

2. Arai T, Hasegawa M, Akiyama $\mathrm{H}$, et al. TDP-43 is a component of ubiquitin-positive tau-negative inclusions in frontotemporal lobar degeneration and amyotrophic lateral sclerosis. Biochem Biophys Res Commun 2006;351:602-11.

3. Neumann M, Sampathu DM, Kwong LK, et al. Ubiquitinated TDP-43 in frontotemporal lobar degeneration and amyotrophic lateral sclerosis. Science 2006;314:130-3.

4. Uchida A, Sasaguri H, Kimura N, et al. Non-human primate model of amyotrophic lateral sclerosis with cytoplasmic mislocalization of TDP-43. Brain 2012:135:833-46.

5. Hasegawa M, Nonaka T, Tsuji $\mathrm{H}$, et al. Molecular dissection of TDP-43 proteinopathies. J Mol Neurosci 2011;45:480-5.
6. Münch C, O'Brien J, Bertolotti A. Prion-like propagation of mutant superoxide dismutase-1 misfolding in neuronal cells. Proc Natl Acad Sci U S A 2011:108:3548-53.

7. Johnson BS, Snead D, Lee JJ, et al. TDP-43 is intrinsically aggregation-prone, and amyotrophic lateral sclerosis-linked mutations accelerate aggregation and increase toxicity. J Biol Chem 2009;284:20329-39.

8. Furukawa Y, Kaneko K, Watanabe $\mathrm{S}$, et al. A seeding reaction recapitulates intracellular formation of Sarkosyl-insoluble transactivation response element (TAR) DNA-binding protein-43 inclusions. J Biol Chem 2011:286:18664-72.

9. Pesiridis GS, Tripathy K, Tanik S, et al. A "two-hit" hypothesis for inclusion formation by carboxyl-terminal fragments of TDP-43 protein linked to RNA depletion and impaired microtubule-dependent transport. J Biol Chem 2011;286:18845-55.

10. Colombrita C, Zennaro E, Fallini C, et al. TDP-43 is recruited to stress granules in conditions of oxidative insult. J Neurochem 2009;111:1051-61.

11. Grad LI, Guest WC, Yanai A, et al. Intermolecular transmission of superoxide dismutase 1 misfolding in living cells. Proc Natl Acad Sci U S A 2011:108:116398-403.

12. Urushitani M, Sik A, Sakurai T, et al. Chromogranin-mediated secretion of mutant superoxide dismutase proteins linked to amyotrophic lateral sclerosis. Nat Neurosci 2006;9:108-18.

13. Urushitani M, Ezzi SA, Julien JP. Therapeutic effects of immunization with mutant superoxide dismutase in mice models of amyotrophic lateral sclerosis. Proc Natl Acad Sci U S A 2007;104:2495-500.

14. Boillée S, Yamanaka K, Lobsiger CS, et al. Onset and progression in inherited ALS determined by motor neurons and microglia. Science 2006;312:1389-92.

15. Yamanaka K, Chun SJ, Boillee S, et al. Astrocytes as determinants of disease progression in inherited amyotrophic lateral sclerosis. Nat Neurosci 2008;11:251-3.

16. Papadeas ST, Kraig SE, O'Banion C, et al. Astrocytes carrying the superoxide dismutase 1 (SOD1G93A) mutation induce wild-type motor neuron degeneration in vivo. Proc Natl Acad Sci U S A 2011;108:17803-8.

17. Haidet-Phillips AM, Hester ME, Miranda CJ, et al. Astrocytes from familial and sporadic ALS patients are toxic to motor neurons. Nat Biotechnol 2011;29:824-8.

18. Brooks BR, Sufit RL, DePaul R, et al. Design of clinical therapeutic trials in amyotrophic lateral sclerosis. In: Rowland LP, ed. Amyotrophic lateral sclerosis and other motor neuron disease. Adv Neurol 1991;56:521-645.

19. Ravits J, Paul P, Jorg C. Focality of upper and lower motor neuron degeneration at the clinical onset of ALS. Neurology 2007;68:1571-5.

20. Körner S, Kollewe K, Fahlbusch M, et al. Onset and spreading patterns of upper and lower motor neuron symptoms in amyotrophic lateral sclerosis. Muscle Nerve 2011;43:636-42.

21. Fujimura-Kiyono C, Kimura F, Ishida S, et al. Onset and spreading patterns of lowe neuron involvements predict survival in sporadic amyotrophic lateral sclerosis. $J$ Neurol Neurosurg Psychiatry 2011:82:1244-9

22. Haverkamp LJ, Appel V, Appel SH. Natural history of amyotrophic lateral sclerosis in a database population. Validation of a scoring system and a model for survival prediction. Brain 1995;118:707-19.

23. Turner MR, Wicks P, Brownstein CA, et al. Concordance between site of onset and limb dominance in amyotrophic lateral sclerosis. J Neurol Neurosurg Psychiatry 2011:82:853-4.

24. Velding JH, Kalmijin S, Groeneveld GJ, et al. Physical activity and the association with sporadic ALS. Neurology 2005;64:241-5

25. Swash M, Leader M, Brown A, et al. Focal loss of anterior horn cells in the cervical cord in motor neuron disease. Brain 1986;109:939-52.

26. Tsukagoshi H, Yanagisawa N, Oguchi K. Morphometric quantification of the cervical limb motor cells in various neuromuscular disease. J Neurol Sci 1980;47:463-72

27. de Carvalho MA, Pinto S, Swash M. Paraspinal and limb motor neuron involvement within homologous spinal segments in ALS. Clin Neurophysiol 2008;119:1607-13.

28. Kuwabara S, Sonoo M, Komori T, et al; Neuromuscular Electrodiagnosis Study Group. Dissociated small hand muscle atrophy in amyotrophic lateral sclerosis: frequency, extent, and specificity. Muscle Nerve 2008;37:426-30.

29. Bae JS, Sawai S, Misawa S, et al. Differences in excitability properties of FDI and ADM motor axons. Muscle Nerve 2009;39:350-4.

30. Pun S, Santos AF, Saxena $S$, et al. Selective vulnerability and pruning of phasic motoneuron axons in motoneuron disease alleviated by CNTF. Nat Neurosci 2006:9:408-19.

31. Dengler R, Konstanzer A, Kuther G, et al. Amyotrophic lateral sclerosis: macro-EMG and twithch forces of single motor units. Muscle Nerve 1990;13:545-50.

32. Ravits JM, La Spada AR. ALS motor phenotype heterogeneity, focality, and deconstructing motor neuron degeneration. Neurology 2009;73:805-11.

33. Ravits JM, Laurie P, Fan Y, et al. Implication of ALS focality: rostral-caudal distribution of lower motor neuron loss postmortem. Neurology 2007;68:1576-82

34. Turner MR, Brockington A, Scaber J, et al. Pattern of spread and prognosis in lower limb-onset ALS. Amyotroph Lateral Scler 2010;11:369-73.

35. Gargiulo-Monachelli GM, Janota F, Bettini M, et al. Regional spread pattern predicts survival in patients with sporadic amyotrophic lateral sclerosis. Eur J Neurol 2012;19:834-41.

36. Shinoda $\mathbf{Y}$, Zarzecki $\mathrm{P}$, Asanuma $\mathrm{H}$. Spinal branching of pyramidal tract neurons in the monkey. Exp Brain Res 1979;34:59-72.

37. Kiernan JA, Hudson AJ. Changes in sizes of cortical and lower motor neurons in amyotrophic lateral sclerosis. Brain 1991;114:843-53. 
38. Pamphlett R, Kril J, Hng TM. Motor neuron disease: a primary disorder of corticomotoneurons? Muscle Nerve 1995; 18:314-18.

39. Attarian S, Vedel JP, Pouget J, et al. Progression of cortical and spinal dysfunctions over time in amyotrophic lateral sclerosis. Muscle Nerve 2008:37:364-75.

40. de Carvalho M, Pinto S, Swash M. Does the motor cortex influence denervation in ALS? EMG studies of muscles with both contralateral and bilateral corticospinal innervation. Clin Neurophysiol 2011;122:629-35.

41. Yokota T, Yoshino A, Inaba A, et al. Double cortical stimulation in amyotrophic lateral sclerosis. J Neurol Neurosurg Psychiatry 1996:61:596-600.
42. Vucic S, Kiernan MC. Novel threshould tracking techniques suggest that cortical hyperexcitability is an early feature of motor neuron disease. Brain 2006;129:2436-46.

43. Vucic S, Nicholson GA, Kiernan MC. Cortical hyperexcitability may precede the onset of familial amyotrophic lateral sclerosis. Brain 2008;131:1540-50.

44. Weber M, Eisen A, Stewart $\mathrm{H}$, et al. The split hand in ALS has cortical basis. J Neurol Sci 2000;180:66-70.

45. Priori A, Oliviero A, Donati $\mathrm{E}$, et al. Human handedness and asymmetry of the motor cortical silent period. Exp Brain Res 1999;128:390-6.

\section{DIFFERENTIAL DIAGNOSIS}

\section{Trustworthy guidance on your iPhone}
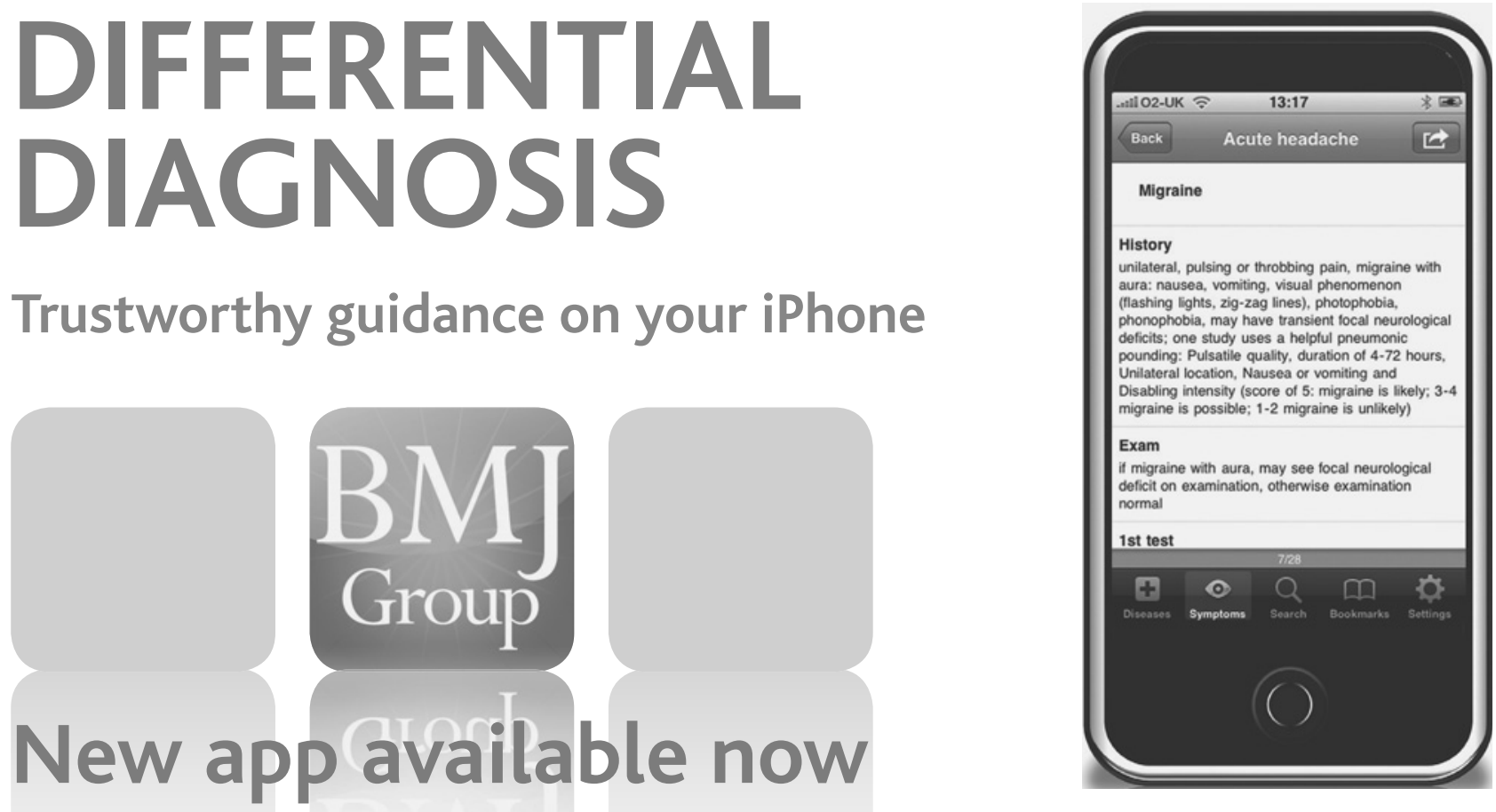

Find out more at bestpractice.bmj.com/differentials 